Michael Trott

\title{
The Mathematica GuideBook for Programming
}

With 315 Illustrations

黛 Springer 


\section{Contents}

0. Introduction to The Mathematica GuideBooks xxi

\section{CHAPTER 1}

\section{Introduction to Mathematica}

1.0 Remarks 1

1.1 Basics of Mathematica as a Programming Language 1

1.1.1 General Background 1

1.1.2 Elementary Syntax 3

1.2 Introductory Examples 7

1.2.0 Remarks 7

1.2.1 Numerical Computations 7

1.2.2 Graphics 31

1.2.3 Symbolic Calculations 54

1.2.4 Programming 80

1.3 What Computer Algebra and Mathematica 4.0

Can and Cannot Do 98

Exercises 102

Solutions 103

References 110

CHAPTER 2

\section{Structure of Mathematica Expressions}

\subsection{Remarks 143}

\subsection{Expressions 143}

2.2 Simple Expressions 147

2.2.1 Numbers and Strings 147

2.2.2 Simplest Arithmetic Expressions and Functions 156

2.2.3 Elementary Transcendental Functions 164 
2.2.4 Mathematical Constants 171

2.2.5 Inverse Trigonometric and Hyperbolic Functions 180

2.2.6 Do Not Be Disappointed 192

2.2.7 Exact and Approximate Numbers 194

2.3 Nested Expressions 197

2.3.1 An Example 197

2.3.2 Analysis of a Nested Expression 200

2.4 Manipulating Numbers 215

2.4.1 Parts of Fractions and Complex Numbers 215

2.4.2 Digits of Numbers 217

Exercises 226

Solutions 230

References 271

CHAPTER 3

\section{Definitions and Properties of Functions}

3.0 Remarks 275

3.1 Defining and Clearing Simple Functions 275

3.1.1 Defining Functions 275

3.1.2 Clearing Functions and Values 292

3.1.3 Applying Functions 297

3.2 Options and Defaults 298

3.3 Attributes of Functions 304

\subsection{Downvalues and Upvalues 317}

3.5 Functions that Remember Their Values $\mathbf{3 2 9}$

3.6 Functions in the $\lambda$-Calculus 336

3.7 Repeated Application of Functions 346

3.8 Functions of Functions 359

Exercises 363

Solutions 368

References 392 
CHAPTER 4

\section{Meta-Mathematica}

\subsection{Remarks 397}

4.1 Information on Commands 397

4.1.1 Information on a Single Command 397

4.1.2 A Program that Reports on Functions 410

4.2 Control over Running Calculations and Resources 416

4.2.1 Intermezzo on Iterators 416

4.2.2 Control over Running Calculations and Resources 422

\subsection{The \$-Commands 425}

4.3.1 System-Related Commands 425

4.3.2 Session-Related Commands 429

4.4 Communication and Interaction with the Outside $\mathbf{4 3 3}$

4.4.1 Writing to Files 433

4.4.2 String Manipulations 438

\subsection{Debugging 442}

4.6 Localization of Variable Names 449

4.6.1 Localization of Variables in Iterator Constructions 449

4.6.2 Localization of Variables in Subprograms 450

4.6.3 Comparison of Scoping Constructs 456

4.6.4 Localization of Variables in Contexts 469

4.6.5 Contexts and Packages 479

4.6.6 Special Contexts and Packages 489

4.7 The Process of Evaluation 499

Exercises 511

Solutions 515

References 536

CHAPTER 5

\section{Restricted Patterns and Replacement Rules}

\subsection{Remarks 539}

\subsection{Boolean Functions $\mathbf{5 3 9}$}

5.1.1 Boolean Functions for Numbers 539

5.1.2 Boolean Functions for General Expressions 549 
5.1.3 Logical Operations 561

5.1.4 Control Structures 563

5.2 Patferns 567

5.2.1 Patterns for Arbitrary Variable Sequences 567

5.2.2 Patterns with Special Properties 580

5.2.3 Attributes of Functions and Pattern Matching 601

\subsection{Replacement Rules 610}

5.3.1 Replacement Rules for Patterns 610

5.3.2 Large Numbers of Replacement Rules 628

5.3.3 Programming with Rules 630

Exercises 644

Solutions 654

References 695

CHAPTER 6

\section{Operations on Lists, and Linear Algebra}

6.0 Remarks 701

6.1 Creating Lists 707

6.1.1 Creating General Lists 707

6.1.2 Creating Special Lists 713

6.2 Representation of Lists 719

6.3 Manipulations on Single Lists 723

6.3.1 Shortening Lists 723

6.3.2 Extending Lists 728

6.3.3 Sorting and Manipulating Elements 729

6.4 Operations with Several Lists or with Nested Lists 750

6.4.1 Simple Operations 750

6.4.2 List of All System Commands 763

6.4.3 More General Operations 777

6.4.4 Constructing a Crossword Puzzle 784

6.5 Mathematical Operations with Matrices 802

6.5.1 Linear Algebra 802

6.5.2 Constructing and Solving Magic Squares 831

6.5.3 Powers and Exponents of Matrices 840 


\subsection{The Top Ten Built-in Commands 849}

Exercises 869

Solutions 885

References 994

Index 1003 